\title{
Rotavirus breakthrough infections responsible for gastroenteritis in vaccinated infants who presented with acute diarrhea at University Teaching Hospitals, Children's Hospital in 2016, in Lusaka Zambia
}

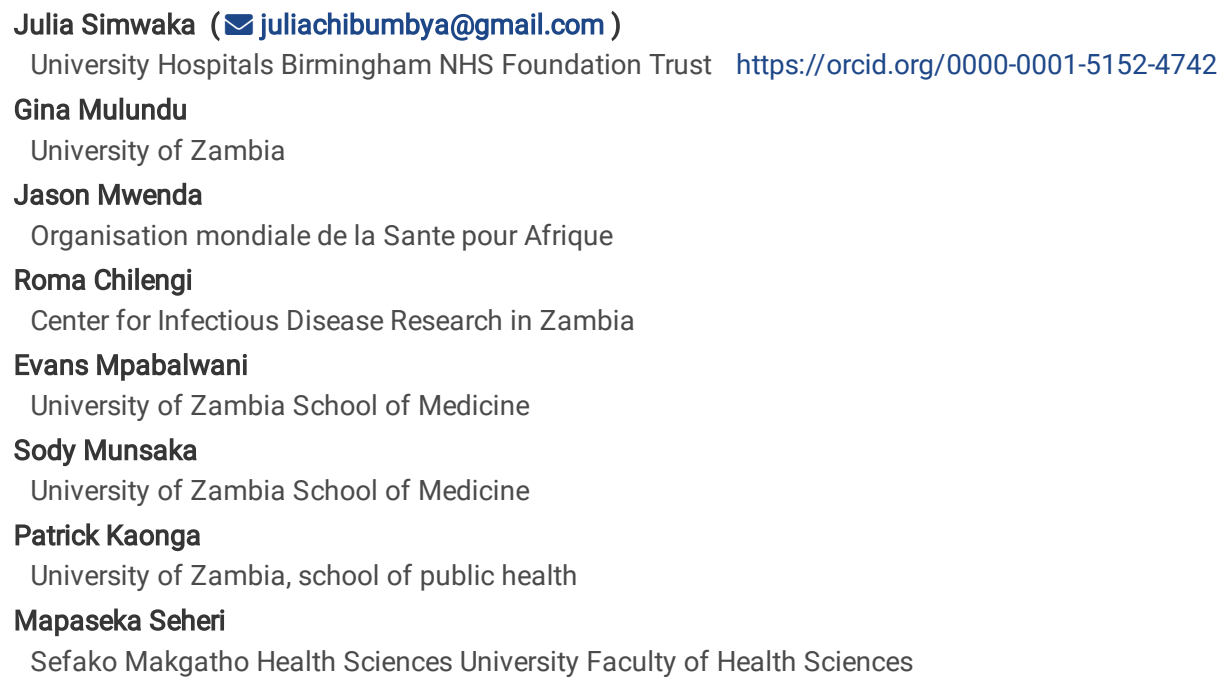




\section{Abstract}

Background Rotavirus has continued to affect under five children despite the introduction of the vaccine. This study aimed at characterizing the rotavirus genotypes responsible for diarrhoea infections in vaccinated infants aged 2 to 12 months and determined the relationship between rotavirus strains and the severity of diarrhoea in 2016.

Materials and Methods We tested stool for group A rotavirus P6 antigen using an ELISA kit (ProSpecT TM , Oxford, UK). All positive specimens with enough sample were genotyped using reverse transcriptase polymerase chain reaction (RT-PCR). A 20-point Vesikari clinical score was used to determine the severity of acute diarrhoea. Diarrhoea scores between 1-5 were considered as mild, 6-10 scores were considered as moderate and scores greater or equal to 11 considered as severe.

Results $153 / 424(36 \%, 95 \% \mathrm{Cl} 31.5 \%$ to $40.9 \%)$ were positive for VP6 rotavirus antigen. The age specific rotavirus infections decreased significantly ( $\mathrm{p}=$ 0.041) from 2 - 4 months $32.0 \%$,followed by a 38.8\% infection rate in the 5-8 months category and subsequently dropped in the infants aged 9-12 months with a positivity rate of $27.2 \% .12 .3 \%$ received a single dose of rotarix vaccine, $77.8 \%$ received 2 doses, and $9.9 \%$ were not vaccinated. $38.5 \%$ of those who received a single dose, $34.5 \%$ of those who received a complete dose and $45.2 \%$ of the unvaccinated tested positive for rotavirus. Infants who tested positive differed significantly from those who tested negative in terms of fever $(p=0.010)$. The predominant rotavirus genotypes were, G2P[6] 36\%, G1P[8] 32\%, mixed infections 19\%, G2P[4] 6\%, G1P[6] 4\% and G9P[6] 3\%. Infants who were infected with G1P8 and G2P[6] had a median Vesikari score of 6, while those who were infected with G1P[6] and mixed infections had a median score of 7 and those with infections due to G2P[4] and G9P[6] had median scores of 4 and 8 respectively.

Discussion and Conclusion The breakthrough infection of heterotypic strains (G2P[6] (36\%), homotypic, G1P[8] (32\%) and mixed infections (19\%) raises concerns about the effects of the vaccination on the rotavirus diversity, considering the selective pressure that rotavirus vaccines could exert on viral populations.

\section{Background}

Rotavirus is the primary cause of acute gastroenteritis in children under five years of age worldwide. Rotavirus diarrhoea is attributed to 215,000 deaths worldwide and 121,000 (56\%) of the deaths occur in sub-Saharan Africa [1].Currently, rotaviruses are associated with 24 million diarrhoeal episodes requiring clinic visits, with an estimated 114 million rotavirus episodes requiring home care per year, and 2.4 million rotaviruses associated hospitalizations globally[2] Diarrhoea is the third leading cause of death after pneumonia and malaria in Zambia. It caused an estimated 10 million episodes in 2015 which resulted in 63,000 hospitalizations and 15,000 deaths, [3]. Because of the high disease burden of rotavirus diarrhoea, WHO recommended the inclusion of either of the two rotavirus vaccines in the routine immunization schedule of each country [4]. Zambian introduced Rotarix ${ }^{\mathrm{T} M}$, a monovalent, live attenuated vaccine which contains a single human G1P [8] rotavirus strain. The two vaccines have also been tested in developing countries and the efficacy is lower as compared to the efficacy in developed countries [5, 6, 7]. The error-prone RNA-dependent RNA polymerase predisposes rotaviruses to genetic mutation and the segmented RNA genome inclines the virus to genome reassortment. These evolutionary mechanisms generate novel strains and have the potential to lead to the emergence of vaccine escape mutants [8]. Reduction and the best prevention measure of severe diarrhoea due to rotaviruses can be attained by using rotavirus vaccines. To fulfil this recommendation, Zambia introduced the live attenuated oral rotavirus vaccine, Rotarix, in its routine immunization schedule in different phases. The first phase was introduction in four districts (Luangwa, Kafue, Chongwe and Chibombo) of Lusaka province in January 2012 and the follow-up phase was the countrywide roll out in November 2013 [9]. Vaccinated children are still presenting with acute diarrhoea and there is need to document the genotypes that are causing acute diarrhoea in these vaccinated infants. This prompted the design of this study to determine strains that were contributing to the cause of diarrhoea in vaccinated infants in 2016. Documenting these possible emerging strains that may be shed after vaccine introduction is important though it is difficult to ascertain the role that the vaccine could have on the emergence and increase of these diverse rotavirus strains, as full genome sequencing was not done to confirm whether they are genetically and structurally different from the one that was used to manufacture the vaccine.

\section{Methods}

Stool samples were selected retrospectively from a pool that were collected for sentinel surveillance for acute gastroenteritis at the University Teaching Hospitals (UTHs), Children`s Hospital in Lusaka, Zambia. Systematic sampling targeted samples from infants aged 2 to 12 months who presented to the hospital with acute diarrhoea of three or more episodes in 24 hours. The stool samples were tested for group A, targeting Viral Protein (VP) 6 antigen using an ELISA kit (ProSpecT ${ }^{T M}$, Oxford, UK). All positive specimens that had enough sample were genotyped using reverse transcriptase polymerase chain reaction (RTPCR). A 20-point Vesikari clinical score was used to determine the severity of acute diarrhoea in infants under study. Diarrhoea scores between 1-5 were considered as mild, 6-10 scores were considered as moderate and scores between 11-20 considered as severe.

Screening for Group A human rotavirus

Out of 823 under five cases that were enrolled for acute diarrhoea surveillance at UTHs Children

shosntal $\in 2016,512 s \rightarrow$ olspecimensweresystematicallyse $\leq$ cted, withoutreplacement, $f$ or thisstudy. Outofthe512s $\rightarrow$ olspecimens, $424(\varepsilon$ s instructions.

Genotyping of EIA positive samples by RT-PCR to determine rotavirus genotypes

Positive stool samples that had enough sample (116 samples, $75.8 \%$ ) were stored at $-20^{\circ} \mathrm{C}$ before being genotyped at Sefako Makgatho Health Sciences University, South African Medical Research Council, Diarrhoeal Pathogens Research Unit and WHO AFRO Rotavirus Regional Reference Laboratory, 
Department of Virology, Pretoria, South Africa, to characterize the rotavirus genotypes. Rotavirus dsRNA extraction was performed from 140 ml of a $10 \%$ fecal suspension prepared by diluting a pea size stool specimen or $100 \mathrm{ul}$ of watery diarrhoea in distilled water. A QIAamp viral RNA mini kit (Qiagen, Hilden,

Germany) was used to extract dsRNA per the manufacturer's instructions. Reverse transcription polymerase chain reaction (RT-PCR) was performed according to previously described protocols complied in the WHO manual of rotavirus detection and characterization methods [4]. The primer pair sBeg/End9 was used to reverse-transcribe the full-length VP7 gene (1062 bp) [10]. While, Con2/Con3 primer set was used to amplify a partial-length of VP4 gene (876 bp) as described by Gentsch et al.[11]. The rotavirus genotyping assays for G-types were carried out using a semi-nested multiplex PCR with type-specific primers (RVG or EndA and aAT8v, aBT1, aCT2, aDT4,mG3, mG9, mG10 and G12) to determine G1, G2, G3, G4, G8, G9,G10 and G12 genotypes [10, 12, 13]. Type-specific primers (dP[8], 2 T-1, 3 T-1, 4 T-1, 5 T-1, P[11] and P[14]) with Con3 were used to determine P[4], P[6], $P[8], P[9], P[10], P[11]$ and $P[14]$ genotypes [11 , 12, 14]. Amplification cycling conditions used were as follows; 30 cycles of denaturation at $94^{\circ} \mathrm{C}$ for 1 min, annealing at $42^{\circ} \mathrm{C}$ for 1 min, extension at $72^{\circ} \mathrm{C}$ for 1 min, with final extension at $72^{\circ} \mathrm{C}$ for $7 \mathrm{~min}$. The reaction was held at $4^{\circ} \mathrm{C}$ until the reaction tubes were removed and electrophoresis was done to visualize the amplicons. A $100 \mathrm{bp}$ molecular weight marker was used to determine the size of the amplicons and classify them accordingly. A negative and positive control was included in all the reactions for both VP7 and VP4 genes. A type-specific nested PCR assay was used to confirm genotypes in mixed infection samples.

\section{Statistical Analysis}

Data was entered using Epi data version 3.1(120306), (Epidata Association, Jens M. Laurisen Michael Bruus, Denmark) and exported to Stata version 13.1 (Stata Corp, College Station, Texas) for final analysis. The comparison of frequencies between positive and negative rotavirus groups was done using proportions. Data were tested for normality using the Shapiro-Francia w test in Stata and was found to be skewed. A 20-point Vesikari clinical score was used to determine the severity of acute diarrhoea in infants under study. Parameters for clinical assessment of diarrhea severity included: duration and maximum number of episodes of diarrhea and vomiting, intensity of fever and dehydration. As per the Vesikari score, [15] a grade of 1-5 was considered as mild, 6-10 as moderate, $11-20$ as severe. The significance was set at a P-value of less than 0.05 .

\section{Results}

The demographic and clinical characteristics of the infants age 2-12 months who were hospitalized for acute gastroenteritis according to their rotavirus status is displayed in Table 1 above. Out of 424 stool samples that were tested, 245 (57.8\%) were male and 179 (42.2\%) were female infants. Out of the 245 males tested for rotavirus diarrhoea, $96(39.2 \%)$ had acute rotavirus diarrhoea, and out of 179 female infants tested, 57 (31.8\%) were infected with rotavirus. Both male and female infants had the same chance of having acute rotavirus diarrhoea $(p=0.126)$.

Overall, there was a significant age-specific association between infants who had rotavirus diarrhoea and those that were negative for rotavirus $(p=0.041)$. The age specific rotavirus infections increased from 2-4 months, 49/118 (41.5\%), peaked in the 5-8 months category 70/181 (38.8\%) and subsequently dropped in the infants aged 9-12 months 34/125 (27.2\%). Infants who had acute rotavirus diarrhoea did not differ significantly from those who did not have rotavirus diarrhoea in the type of treatment that they received $(p=0.123)$, even though the majority of infants who presented to the hospital with acute diarrhoea received intravenous fluid 238/424 (56.1\%).

Infants who had rotavirus acute diarrhoea were not significantly different from those that did not have rotavirus diarrhoea in the number of episodes of diarrhoea ( $p=0.969)$, episodes of vomiting $(P=0.219)$, duration of diarrhoea and vomiting, $(P=0.402$. and 0.188 respectively). Infants who received 1 dose or 2 doses and those not vaccinated at all had the same chance of having acute diarrhoea due to rotavirus $(p=0.351)$. Infants who had acute rotavirus gastroenteritis differed significantly from those who were negative for rotavirus by fever, $(P=0.010)$. The median duration of acute diarrhoea in days was 3 days (IQR, 2-3), and the median episodes of diarrhoea in 24 hours were 5 episodes (IQR, 4-6). The median duration of vomiting was 2 days (IQR, 1-3) while the median episodes of vomiting in 24 hours were 3 episodes (IQR, 2-4).

Figure 1 below shows the age distribution of rotavirus genotypes which were responsible for the acute diarrhoea that was seen in infants who presented with acute diarrhoea at UTHs Children's Hospital. Overall, there was no significant difference in the genotypes that infected infants aged 2-12 months who presented with acute diarrhoea in 2016. Most infants aged 2-4 months had acute diarrhoea due to G2P[6] 15/42 (35.7\%) followed by G1P[8] 11/37 (29.7\%). $4 / 22(18.2 \%)$ of rotavirus infections that occurred in this age group were due to mixed genotype infections. A few infections were caused by G2P[4] 4/7 and G9P[6] 3/4. There was no G1P[6] infections in this age group. Infants age 5-8 months were infected with all rotavirus genotypes which were detected in this study with most infections being caused by G2P[6] 20/42 (47.6\%), followed by G1P[8] 19/37 (51.4\%) and mixed infections 13/22 (59.1\%). The older age group, 9-12 months were infected with G1P[8] 7/37 (18.9\%), G2P[6] 7/42 (16.7\%), and mixed infections 5/22 (22.7\%).

Out of 424 samples which were eventually tested for human group A rotavirus VP6 antigen, 153 stool samples, representing $36.1 \%$, (95\% Cl $31.5 \%$ to $40.9 \%)$ were positive for rotavirus VP6 antigen and 271 (64\%) were negative. Rotavirus acute diarrhoea occurred throughout the year with highest pick occurring in the cool dry months through to the dry hot months of the year, as shown in the graph below.

Most rotavirus infections which occurred in infants in this study were because of G2P[6] 42/116 (36 \%), followed by G1P[8] 37/116 (32\%), mixed infections 22/116 (19\%). Rotavirus genotypes that were responsible for the dual infections that occurred in the infants who were studied included, G9P[6]/P[8], G2P[6]/P[4], G1P[8]/P[4]/P[6], G1/G2P[6], G1P[4]/P[8], G-P[8]/P[4], G8/G3P[6], G3/G9P[6], G1P[6]/P[8], G1/G2P[4], G2/G9 P[6]/P[8]G2 P[6]/P[4] G2/G9 P[6], G9P[6]/P[8], G1/G9P[6],G1/G12P[6]. G2P[4] was only responsible for 7/116 (6\%), G1P[6] 4/116 (4\%.), and a few G9P[6] 4/116 (3\%) as indicated in Figure 3 below. Figure 4 below shows that monthly variation of acute diarrhoea infections which occurred in infants aged 2-12 months at UTHs Children's Hospital in 2016. Many infections which occurred in January 2016 where as a results of G2P [6] 8/22 (36.4\%); followed by G1P[8] 6/22 (27.3\%); followed by G2P[4] 4/22 $(18.2 \%)$ and a few mixed infections $3 / 22(18.2 \%)$. There was a reduction in acute diarrhoea cases due to rotavirus in February through to May 2016 , with a few infections that occurred in these months being caused by G1P[8], mixed infections and G2P[6]. The majority of acute diarrhoea infections which occurred 
in June (coolest month in Zambia) where as a results of G1P[8] 16/23 (69.6\%), followed by G2P[6] 3/23(13.0\%), mixed infections 3/23 (13.0\%) and one infection was due G2P[4] 1/23 (4.4\%).

The greater part of infections that occurred in July were due to G1P[8] 4/8 (50.0 \%), followed by G2P[6] 2/8 (25.0\%), and mixed infections 2/8 (25.0\%). In August, eleven acute diarrhoea infections which occurred in this month were as a result of G2P[6] 4/11 (36.4\%), followed by G1P[8] 3/11 (27.3\%), mixed infections 3/11 (27.3\%), and one infection which was caused by G1P[6] strain. September is the beginning of the hot dry season in Zambia and that is where most rotavirus infections $33 / 116$ took place. The common genotype which caused acute diarrhoea in infants who presented to the hospital in this month was G2P[6] 23/33(69.7\%). The rest of the acute rotavirus diarrhoeas which occurred in September were due to mixed infections 7/33 (21.2\%), G2P[4 2/33 (6.1\%) and G1P[8] 1/33 (3.0\%). There was a reduction of acute diarrhoea due to rotavirus in the months of October through to December in 2016 , with the a few rotavirus acute diarrhoea infections being caused by G1P[8], G1P[6], G9P[6], G2P[6] and mixed infections.

As shown in figure 5 below, most rotavirus acute diarrhoea infections which occurred in 2016 were mild to moderate. The median Vesikari score for acute diarrhoeal infections the occurred due to G1P[8] and G2P[6] was 6, indicating moderate infections with Vesikari score between 6-10. The median Vesikari score for infections which were due G1P[6] and mixed infections was 7, causing moderate acute diarrhoea with Vesikari scores ranging between 6-10, while 4 and 8 were the median Vesikari scores for infections caused by G2P[4] and G9P[6] respectively. This data shows that most of the acute diarrhoea infections which occurred in 2016 were mild to moderate infections; we did not record any severe diarrhoea except for 3 cases which were because of G2P[6] and had a Vesikari score of 11 .

\section{Discussion}

This study demonstrates that slightly over a third (36.1\%) of infants aged 2-12 months who were admitted at the UTHs Children's Hospital due to acute diarrhoea had rotavirus infection. This prevalence is slightly higher than the one reported in another study carried out in Lusaka by Mpabalwani and colleagues, who found the prevalence of rotavirus acute diarrhoea to be $24.7 \%$ in 2014 and $27 \%$ in 2015 [16]. This observation may be due to the difference in the study participants and the period of analysis. In the previous study, all under five children were analysed over a period of over 2 years, while this study looked at infants aged 2-12 months over a period of 12 months. A study conducted in Rwanda documented a decline in the proportion of hospitalizations due to rotavirus diarrhoea among children $<1$ year of age [17]. Another study conducted in Zimbabwe reported a rotavirus disease burden of $25 \%$ in under five children after the vaccine was introduced [18]. A study conducted in Kenya observed a prevalence of rotavirus diarrhoea of $14.5 \%$ after the vaccine was introduced in their routine immunization schedule [19]. These variations may reflect actual differences in rotavirus gastroenteritis disease burden, but may also be related to variations in study design, the methods used in detecting rotavirus, and vaccination against rotavirus diarrhoea.

This study demonstrates that there is an age specific significant difference in the burden of rotavirus infection in the infants who were studied $(p=0.041)$. These findings are consistent with the study that was conducted in Nepal where they found that the highest prevalence of rotavirus diarrhoea occurred in infants aged $0-11$ months $(26.7 \%)$ followed by $23.6 \%$ in the $12-23$ months [20].

Rotavirus infections in 2016 occurred throughout the year, with the highest peak being in June, 52\% (cool dry month), September and October (hot dry months) $57 \%$ and $52 \%$ respectively. These findings are consistent with the findings documented in a study conducted in Nigeria where they had a marked seasonal peak, with more than $90 \%$ of rotavirus infections occurring during December to March, their cool dry months [21]. Similar results were documented from a study conducted by Mwenda and colleagues who showed that rotavirus infections occurred throughout the year in other African countries (Uganda, Ghana, and Ethiopia), with a peak prevalence generally being recorded during the cool dry months of the year [22].

A diversity of rotavirus genotypes, G2P[6] 36\%, G1P[8] 32\%, mixed infections 19\%, G2P[4] 6\%, G1P[6] 4\%, and G9P[6] 3\% were predominant in 2016. These findings are consistent with the findings of Seheri and colleagues who found that the predominant genotypes circulating in sub-Saharan Africa included G1P[8] (23.8\%), followed by G2P[4] (11.8\%), G9P[8] (10.4\%), G12P[8] (4.9\%), G2P[6] (4.2\%) and G3P[6] (3.7\%) [23]. The findings are also consistent with the report of the African Rotavirus Surveillance Network among hospitalized children aged $<5$ years which showed a high diversity of circulating rotavirus strains in the subcontinent [22]. According to this study findings, G2P[6], G1P[8] and mixed infections caused most of the acute diarrhoea infections which occurred in 2016. These breakthrough infections reached their highest peaks in January, June and September with G2P[6] being predominant in January and September and G1P[8] in June. Rotarix, a monovalent rotavirus vaccine is expected to provide cross protection against the circulating rotavirus genotypes that share either the $\mathrm{G}$ or the P type with the vaccine strain G1P[8] (homotypic protection)[24]. In this study, $77.8 \%$ of infants who presented with rotavirus diarrhoea received a complete dose ( 2 doses), $12.3 \%$ received incomplete ( 1 dose) and $9.9 \%$ were not vaccinated. It is important to note that most rotavirus infections which occurred in fully vaccinated infants were caused by G2P[6] (38.4\%), followed by G1P[8] $27.9 \%$ and mixed infections $19.8 \%$. There is need to do a full genome analysis of G1P[8] to establish whether infection being caused by this genotype are wild or vaccine derived. It is also cardinal to continuously monitor the presence of $\mathrm{G} 2 \mathrm{P}[6]$ to determine whether the increase in this genotype which is seemingly evading the vaccine immunity is due to vaccine pressure or genotype evolution. Vaccine trial studies conducted in Malawi and South Africa in 2012 indicated that a significant protection was afforded by the vaccine against severe gastroenteritis caused by, G2P[4], a heterotypic rotavirus strain (vaccine efficacy against G2: 79.2\% [95\% Cl: 8.9\%; 96.5\%; 0.017 ; vaccine efficacy against P[4]: $70.9 \%$ [95\% Cl: $37.5 \% ; 87.0 \%]$ [24]. There is need to do a strain specific vaccine effectiveness study to confirm the efficacy of the Rotarix vaccine against $P[6]$ since there is limited data concerning the vaccine efficacy of $P[6]$ vaccine in Africa.

The G2P[6], a fully heterotypic genotype to the Rotarix vaccine, infections started increasing after the vaccine was introduced in Lusaka in 2012 , and became predominant throughout the post-vaccine period as indicated in another study [25]. These changes in circulating genotypes after vaccine introduction are consistent with what has been observed in other countries $[26,27,28,29,30,31]$.

G2P[6] strains have a different overall genomic RNA constellation and hence belong to a different genogroup (DS-1) than the Rotarix vaccine strain which belongs to Wa-like genogroup [32]. Since rotarix is a single live attenuated monovalent human G1P[8] strain vaccine, the continued break through infections 
caused by G1P[8] genotype also raises concerns and warrant further investigations by doing a full genome analysis to rule out vaccine derived acute diarrhoea in infants who are vaccinated with Rotarix vaccine

Other studies have linked rotavirus susceptibilities to Histo-Blood Group Antigens (HBGA) phenotype of the host [33, 34]. Depending on the P genotype (either $\mathrm{P}$ [4], P [6] and P [8]), HBGA binding is different. Non- secretor individuals have an active a 1,2 fructosyltransferase and they do not synthesize the H-type 1 antigen thus they are not susceptible to $\mathrm{P}[6]$ rotavirus genotype infections. $\mathrm{P}$ [4] and $\mathrm{P}$ [8] bind specifically by recognizing both the common Le $\mathrm{e}^{\mathrm{b}}$ as the $\mathrm{H}$-type 1 antigen while $\mathrm{P}[6]$ genotypes completely recognize the $\mathrm{H}$-type 1 [35]. These findings from other studies warrant further investigation of Zambia's under five population to investigate the type of secretor status that is common given the substantial amount of G2 P [6] infections in the present study. The finding would also highlight how much of low vaccine efficacy observed in Africa is attributable to the secretor status phenomenon.

These findings are slightly different with what was found in Nicaragua, where it was found that G12 genotype was responsible for most of the diarrhoea hospitalization that they recorded in under five children after the vaccine was introduced in Nicaragua [36]. In Colombia, a study conducted by Pelaez-Carvajal and colleagues demonstrated that G2P [4], a strain fully heterotypic to the Rotarix vaccine, was predominant before vaccine introduction in 2008 (47\%) and after vaccine introduction in 2010 , G2P [4] was responsible for $54 \%$ of diarrhoeal infections observed in infants in the same year, $86 \%$ in 2011 and $32 \%$ in 2012 [37]. Another study conducted by Paulke-Korinek and colleagues demonstrated that G2P [4] genotype caused most of the breakthrough infection in vaccinated children in Austria [38] (Paulke-Korinek, et al., 2013).

The presence of G2P [6], before the introduction of vaccine, although at a lower rate (5\%) [25], and its prevalence after the vaccine was introduced may suggest secular variation rather than vaccine pressure as a cause for the fluctuation. Pelaez-Carvajal and colleagues suggested that surveillance data should be mixed with information on disease incidence and strain specific vaccine effectiveness to avoid misinterpretation about the role of vaccine pressure on emergence of new or persistent strains [37]

\section{Conclusion}

The breakthrough infection of heterotypic strains (G2P[6] (36\%), homotypic, G1P[8] (32\%) and mixed infections (19\%) raises concerns about the effects of the vaccination on the rotavirus diversity, considering the selective pressure that rotavirus vaccines could exert on viral populations. There is need to do a full genome analysis of $\mathrm{G} 1 \mathrm{P}[8]$ to rule out the possibility of vaccine-derived G1P[8] infections, considering that the Rotarix vaccine being used in the routine immunisation schedule is a G1P[8] attenuated monovalent vaccine

\section{Abbreviations}

WHO/ AFRO: World Health Organisation for the African Region

RT-PCR : Reverse Transcriptase Polymerase Chain Reaction

VP 6 : Viral Protein 6 for rotavirus

UTH : University Teaching Hospital

EIA : Enzyme Immunoassay

dsRNA : double stranded Ribonucleic Acid

UNZABREC : University of Zambia Biomedical Research Ethical Committee

IQR : Interquatile range

HBGA : Histo blood Group Antigens

\section{Declarations}

\section{Ethical approval and consent to participate}

Ethical approval was granted by the University of Zambia Biomedical Research Ethical Committee (UNZABREC) when they granted a waiver and permission to conduct this study using stool samples which were collected for surveillance purposes in 2016 (reference number for this approval is 067-06-17; assurance number FWA00000338 IRB00001131 of IORG0000774). There was no direct involvement of study participants, therefore no consent was obtained from parents because only stool samples which where collected for surveillance in 2016 were used for this study.

\section{Consent for publication}

Permission to conduct this study at the virology laboratory and to use the collected surveillance samples for 2016 was also sought from Ministry of Health and University Teaching Hospital management. Data was secured to prevent unauthorized access and no personal identifiers were used during data collection and analysis.

\section{Availability of data and materials}


The data that support the findings of this study are available from Ministry of Health, but restrictions apply to the availability of these data, which were used with permission for the current study, and so are not publicly available. Data are however available from the corresponding author upon reasonable request and with permission of Ministry of Health.

\section{Competing interests}

The authors have no competing interests

\section{Funding}

Financial support for test kits used to test samples come from WHO and the organisation did not play any role in the design of the study, collection, analysis, and interpretation of data and in writing the manuscript.

\section{Authors' contributions}

JS : Played a role in overall study design, data review, interpretation of results and review and drafting of manuscript,

RC : Played a significant role in the overall reviewing of the manuscript, management, data review, interpretation of results

PK: Played a very important role in advising with suitable statistaical packages to use in theanalysis of data and interpretation of results

SM: Played a role designing the study, supervision and management of study

GM: Ensured that the gramma and structure of the manuscript is correct.

EM: Ensured that the whole manuscript is scientifically oriented and the graphical presentation of the paper is correct.

JM: Ensured that the kits and materials that we required to test these samples were provided and he also played a critical role in seeking permission from WHO for the samples to be tested at the regional reference lab in South Africa.

MS: Played an important role in supervising the genotyping work in laboratory to ensure that the results that were produced where of quality and acceptable.All authors read and approved the final manuscript.

\section{Acknowledgements}

We would like to thank you the Sefako Makgatho Health Sciences University, South African Medical Research Council, Diarrhoeal Pathogens Research Unit, WHO AFRO Rotavirus Regional Reference Laboratory, Department of Virology, Pretoria, South Africa, staff members for technical support during the testing of samples and characterizing all the samples used in this study. Special gratitude goes to WHO AFRO for providing financial assistance and provision of testing kits and reagents. We sencirely thank the Ministry of Health and University teaching Hospital management for allowing us to use the surveillance stool samples for this study, including the Virology Unit Head for granting us permission to conduct this study in the virology laboratory.

\section{References}

1. World Health Organization. Global, regional, and national estimates of rotavirus mortality in children< 5 years of age, 2000-2013. Clin Infect Dis. 2016;62(Suppl 2):S96-105.

2. Ali Z, Harastani H, Hammadi M, Reslan L, Ghanem S, Hajar F, Sabra A, Haidar A, Inati A, Rajab M, Fakhouri H. Rotavirus genotypes and vaccine effectiveness from a sentinel, hospital-based, surveillance study for three consecutive rotavirus seasons in lebanon. PloS one. 2016 Aug 29;11(8):e0161345.

3. Chilengi R, Rudd C, Bolton C, Guffey B, Masumbu PK, Stringer J. Successes, challenges and lessons learned in accelerating introduction of rotavirus immunisation in Zambia. World Journal of Vaccines. 2015 Jan 20;5(01):43.

4. World Health Organization. Generic portocols for (i) hospital-based surveillance to estimate the burden of rotavirus gastroenteritis in children and (ii) a community-based survey on utilization of health care services for gastroenteritis in children: field test version. Geneva: World Health Organization; 2002.

5. Armah GE, Sow SO, Breiman RF, Dallas MJ, Tapia MD, Feikin DR, Binka FN, Steele AD, Laserson KF, Ansah NA, Levine MM. Efficacy of pentavalent rotavirus vaccine against severe rotavirus gastroenteritis in infants in developing countries in sub-Saharan Africa: a randomised, double-blind, placebocontrolled trial. The Lancet. 2010 Aug 21;376(9741):606-14.

6. Steele AD, Neuzil KM, Cunliffe NA, Madhi SA, Bos P, Ngwira B, Witte D, Todd S, Louw C, Kirsten M, Aspinall S. Human rotavirus vaccine Rotarix ${ }^{\mathrm{TM}}$ provides protection against diverse circulating rotavirus strains in African infants: a randomized controlled trial. BMC infectious diseases. 2012 Dec;12(1):213.

7. Cunliffe NA, Witte D, Ngwira BM, Todd S, Bostock NJ, Turner AM, Chimpeni P, Victor JC, Steele AD, Bouckenooghe A, Neuzil KM. Efficacy of human rotavirus vaccine against severe gastroenteritis in Malawian children in the first two years of life: a randomized, double-blind, placebo controlled trial. Vaccine. 2012 Apr 27;30:A36-43

8. Jere KC, Chaguza C, Bar-Zeev N, Lowe J, Peno C, Kumwenda B, Nakagomi O, Tate JE, Parashar UD, Heyderman RS, French N. Emergence of double-and triple-gene reassortant G1P [8] rotaviruses possessing a DS-1-like backbone after rotavirus vaccine introduction in Malawi. Journal of virology. 2018 Feb 1;92(3):e01246-17. 
9. Beres LK, Tate JE, Njobvu L, Chibwe B, Rudd C, Guffey MB, Stringer JS, Parashar UD, Chilengi R. A preliminary assessment of rotavirus vaccine effectiveness in Zambia. Clinical Infectious Diseases. 2016 Apr 7;62(suppl_2):S175-82.

10. Gouvea V, Glass RI, Woods P, Taniguchi K, Clark HF, Forrester B, Fang ZY. Polymerase chain reaction amplification and typing of rotavirus nucleic acid from stool specimens. Journal of clinical microbiology. 1990 Feb 1;28(2):276-82.

11. Gentsch JR, Glass RI, Woods P, Gouvea V, Gorziglia M, Flores J, Das BK, Bhan MK. Identification of group A rotavirus gene 4 types by polymerase chain reaction. Journal of clinical microbiology. 1992 Jun 1;30(6):1365-73.

12. Iturriza-Gómara M, Kang G, Gray J. Rotavirus genotyping: keeping up with an evolving population of human rotaviruses. Journal of clinical virology. 2004 Dec 1;31(4):259-65.

13. Aladin F, Nawaz S, Iturriza-Gómara M, Gray J. Identification of G8 rotavirus strains determined as G12 by rotavirus genotyping PCR: updating the current genotyping methods. Journal of Clinical Virology. 2010 Apr 1;47(4):340-4.

14. Rahman M, Sultana R, Ahmed G, Nahar S, Hassan ZM, Saiada F, Podder G, Faruque AS, Siddique AK, Sack DA, Matthijnssens J. Prevalence of G2P [4] and G12P [6] rotavirus, Bangladesh. Emerging infectious diseases. 2007 Jan;13(1):18.

15. Vesikari T, Karvonen A, Prymula R, Schuster V, Tejedor JC, Cohen R, Meurice F, Han HH, Damaso S, Bouckenooghe A. Efficacy of human rotavirus vaccine against rotavirus gastroenteritis during the first 2 years of life in European infants: randomised, double-blind controlled study. The Lancet. 2007 Nov 24;370(9601):1757-63.

16. Mpabalwani EM, Simwaka CJ, Mwenda JM, Mubanga CP, Monze M, Matapo B, Parashar UD, Tate JE. Impact of rotavirus vaccination on diarrheal hospitalizations in children aged< 5 years in Lusaka, Zambia. Clinical Infectious Diseases. 2016 Apr 7;62(suppl_2):S183-7.

17. Sibomana H, Rugambwa C, Mwenda JM, Sayinzoga F, Iraguha G, Uwimana J, Parashar UD, Tate JE. Impact of routine rotavirus vaccination on all-cause and rotavirus hospitalizations during the first four years following vaccine introduction in Rwanda. Vaccine. 2018 Nov 12;36(47):7135-41.

18. Mukaratirwa A, Berejena C, Nziramasanga P, Ticklay I, Gonah A, Nathoo K, Manangazira P, Mangwanya D, Marembo J, Mwenda JM, Weldegebriel G. Distribution of rotavirus genotypes associated with acute diarrhoea in Zimbabwean children less than five years old before and after rotavirus vaccine introduction. Vaccine. 2018 Nov 12;36(47):7248-55.

19. Muendo C, Laving A, Kumar R, Osano B, Egondi T, Njuguna P. Prevalence of rotavirus infection among children with acute diarrhoea after rotavirus vaccine introduction in Kenya, a hospital cross-sectional study. BMC pediatrics. 2018 Dec;18(1):323.

20. Sherchand JB, Thakali O, Sherchan JB, Bhandari D, Tandukar S, Paudel KP, Shrestha BM, Rayamajhi A, Rai GK. Hospital based surveillance and molecular characterization of rotavirus in children less than 5 years of age with acute gastroenteritis in Nepal. Vaccine. 2018 Dec 14;36(51):7841-5.

21. Tagbo BN, Mwenda JM, Eke CB, Edelu BO, Chukwubuike C, Armah G, Seheri ML, Isiaka A, Namadi L, Okafor HU, Ozumba UC. Rotavirus diarrhoea hospitalizations among children under 5 years of age in Nigeria, 2011-2016. Vaccine. 2018 Dec 14;36(51):7759-64.

22. Mwenda JM, Ntoto KM, Abebe A, Enweronu-Laryea C, Amina I, Mchomvu J, Kisakye A, Mpabalwani EM, Pazvakavambwa I, Armah GE, Seheri LM. Burden and epidemiology of rotavirus diarrhea in selected African countries: preliminary results from the African Rotavirus Surveillance Network. Journal of Infectious Diseases. 2010 Sep 1;202(Supplement_1):S5-11

23. Seheri LM, Magagula NB, Peenze I, Rakau K, Ndadza A, Mwenda JM, Weldegebriel G, Steele AD, Mphahlele MJ. Rotavirus strain diversity in Eastern and Southern African countries before and after vaccine introduction. Vaccine. 2018 Nov 12;36(47):7222-30.

24. Steele AD, Neuzil KM, Cunliffe NA, Madhi SA, Bos P, Ngwira B, Witte D, Todd S, Louw C, Kirsten M, Aspinall S. Human rotavirus vaccine Rotarix ${ }^{T M}$ provides protection against diverse circulating rotavirus strains in African infants: a randomized controlled trial. BMC infectious diseases. 2012 Dec;12(1):213.

25. Simwaka JC, Mpabalwani EM, Seheri M, Peenze I, Monze M, Matapo B, Parashar UD, Mufunda J, Mphahlele JM, Tate JE, Mwenda JM. Diversity of rotavirus strains circulating in children under five years of age who presented with acute gastroenteritis before and after rotavirus vaccine introduction, University Teaching Hospital, Lusaka, Zambia, 2008-2015. Vaccine. 2018 Nov 12;36(47):7243-7

26. Carvalho-Costa FA, de Mello Volotão E, de Assis RM, Fialho AM, de Andrade JD, Rocha LN, Tort LF, da Silva MF, Gómez MM, de Souza PM, Leite JP. Laboratory-based rotavirus surveillance during the introduction of a vaccination program, Brazil, 2005-2009. The Pediatric infectious disease journal. 2011 Jan 1;30(1):S35-41.

27. Hull JJ, Teel EN, Kerin TK, Freeman MM, Esona MD, Gentsch JR, Cortese MM, Parashar UD, Glass RI, Bowen MD. United States rotavirus strain surveillance from 2005 to 2008: genotype prevalence before and after vaccine introduction. The Pediatric infectious disease journal. 2011 Jan 1;30(1):S42-7.

28. Kirkwood CD, Boniface K, Barnes GL, Bishop RF. Distribution of rotavirus genotypes after introduction of rotavirus vaccines, Rotarix ${ }^{\circledR}$ and RotaTeq ${ }^{\circledR}$, into the National Immunization Program of Australia. The Pediatric infectious disease journal. 2011 Jan 1;30(1):S48-53.

29. Afrad MH, Hassan Z, Farjana S, Moni S, Barua S, Das SK, Faruque AS, Azim T, Rahman M. Changing profile of rotavirus genotypes in Bangladesh, 20062012. BMC infectious diseases. 2013 Dec;13(1):320.

30. Tanaka T, Kamiya H, Asada K, Suga S, Ido M, Umemoto M, Ouchi K, Ito H, Kuroki H, Nakano T, Taniguchi K. Changes in rotavirus genotypes before and after vaccine introduction: a multicenter, prospective observational study in three areas of Japan. Japanese journal of infectious diseases. 2017:JJID2016

31. Vizzi E, Piñeros OA, Oropeza MD, Naranjo L, Suárez JA, Fernández R, Zambrano JL, Celis A, Liprandi F. Human rotavirus strains circulating in Venezuela after vaccine introduction: predominance of G2P [4] and reemergence of G1P [8]. Virology journal. 2017 Dec;14(1):58.

32. Matthijnssens J, Ciarlet M, Heiman E, Arijs I, Delbeke T, McDonald SM, Palombo EA, Iturriza-Gómara M, Maes P, Patton JT, Rahman M. Full genome-based classification of rotaviruses reveals a common origin between human Wa-Like and porcine rotavirus strains and human DS-1-like and bovine rotavirus strains. Journal of virology. 2008 Apr 1;82(7):3204-19. 
33. Hu L, Crawford SE, Czako R, Cortes-Penfield NW, Smith DF, Le Pendu J, Estes MK, Prasad BV. Cell attachment protein VP8* of a human rotavirus specifically interacts with A-type histo-blood group antigen. Nature. 2012 May;485(7397):256.

34. Huang P, Xia M, Tan M, Zhong W, Wei C, Wang L, Morrow A, Jiang X. Spike protein VP8* of human rotavirus recognizes histo-blood group antigens in a type-specific manner. Journal of virology. 2012 May 1;86(9):4833-43.

35. Heylen E, Zeller M, Ciarlet M, Lawrence J, Steele D, Van Ranst M, Matthijnssens J. Human P [6] rotaviruses from sub-Saharan Africa and Southeast Asia are closely related to those of human P [4] and P [8] rotaviruses circulating worldwide. The Journal of infectious diseases. 2016 Jul 28;214(7):1039-49.

36. Bucardo F, Mercado J, Reyes Y, González F, Balmaseda A, Nordgren J. Large increase of rotavirus diarrhoea in the hospital setting associated with emergence of G12 genotype in a highly vaccinated population in Nicaragua. Clinical Microbiology and Infection. 2015 Jun 1;21(6):603-e1.

37. Peláez-Carvajal D, Cotes-Cantillo K, Paternina-Caicedo A, Gentsch J, de la Hoz-Restrepo F, Patel M. Characterization of rotavirus genotypes before and after the introduction of a monovalent rotavirus vaccine in Colombia. Journal of medical virology. 2014 Jun;86(6):1083-6.

38. Paulke-Korinek M, Kollaritsch H, Aberle SW, Zwazl I, Schmidle-Loss B, Vécsei A, Kundi M. Sustained low hospitalization rates after four years of rotavirus mass vaccination in Austria. Vaccine. 2013 May 31;31(24):2686-91.

\section{Table}

Table 1: Demographic and clinical characteristics of infants who presented with acute diarrhoea at UTHs, Children`s Hospital

\begin{tabular}{|c|c|c|c|c|}
\hline & Total (n-424) & Positive & Negative & p value \\
\hline \multicolumn{5}{|l|}{ Sex } \\
\hline Male & $245(57.8)$ & $96(39.2)$ & $149(60.8)$ & \multirow[t]{2}{*}{0.126} \\
\hline Female & $179(42.2)$ & $57(31.8)$ & $122(68.2)$ & \\
\hline \multicolumn{5}{|l|}{ Age } \\
\hline 2-4 months & 118(27.8) & $49(41.5)$ & $69(58.5)$ & \multirow[t]{3}{*}{0.041} \\
\hline 5-8 months & 181(42.7) & $70(38.8)$ & $111(61.3)$ & \\
\hline 9-12 months & 125(29.5) & $34(27.2)$ & 91(72.8) & \\
\hline \multicolumn{5}{|l|}{ Treatment } \\
\hline Oral rehydration salts (ORS) & $122(28.8)$ & $35(28.7)$ & $87(71.3)$ & \multirow[t]{3}{*}{0.123} \\
\hline Intravenous Fluid (IVF) & $238(56.1)$ & $94(39.5)$ & $144(60.5)$ & \\
\hline ORS+IVF & $64(15.1)$ & $24(37.5)$ & $40(62.5)$ & \\
\hline \multicolumn{5}{|l|}{ Rotavirus vaccine received } \\
\hline 1 Dose & $52(12.3)$ & $20(38.5)$ & $32(61.5)$ & \multirow[t]{3}{*}{0.351} \\
\hline 2 Doses & $330(77.8)$ & $114(34.5)$ & $216(65.5)$ & \\
\hline Unvaccinated & $42(9.9)$ & $19(45.2)$ & $23(54.8)$ & \\
\hline \multicolumn{5}{|l|}{ Duration of Diarrhoea in days } \\
\hline 1-4days & $385(90.8)$ & $141(36.6)$ & $244(63.4)$ & \multirow[t]{3}{*}{0.402} \\
\hline 5 days & $27(6.4)$ & $10(37.0)$ & $17(63.0)$ & \\
\hline$>6$ days & $12(2.8)$ & $2(16.8)$ & $10(83.3)$ & \\
\hline \multicolumn{5}{|l|}{ Diarrhoea episodes in 24 hours } \\
\hline $3-5$ episodes & $307(72.4)$ & $110(35.8)$ & $197(64.2)$ & \multirow[t]{3}{*}{0.969} \\
\hline 6-10 episodes & $112(26.4)$ & $41(36.6)$ & $71(63.4)$ & \\
\hline$>=11$ Episodes & $5(1.2)$ & $2(40.0)$ & $3(60.0)$ & \\
\hline \multicolumn{5}{|l|}{ duration of vomiting in days } \\
\hline No Vomiting & $185(43.6)$ & $70(37.8)$ & $115(62.2)$ & \multirow{4}{*}{0.188} \\
\hline 1 day & $60(14.2)$ & $16(26.7)$ & $44(73.3)$ & \\
\hline 2 days & $89(21.0)$ & $38(42.7)$ & $51(57.3)$ & \\
\hline 3-6 days & $90(21.2)$ & $29(32.2)$ & $61(67.8)$ & \\
\hline \multicolumn{5}{|l|}{$\begin{array}{l}\text { Vomiting episodes in } \\
24 \text { hours }\end{array}$} \\
\hline No vomiting & $185(43.6)$ & $70(37.8)$ & $115(62.2)$ & \\
\hline 1 emesis & $43(10.1)$ & $18(41.9)$ & $25(58.1)$ & \multirow[t]{3}{*}{0.219} \\
\hline $2-4$ emeses & $116(27.4)$ & $33(28.5)$ & $83(71.5)$ & \\
\hline$>5$ emeses & $80(18.9)$ & $32(40.0)$ & $48(60.0)$ & \\
\hline \multicolumn{5}{|l|}{ Fever (Temperature) } \\
\hline $37-38.4 \square c$ & $306(72.2)$ & 101(33.0) & $205(67.0)$ & \multirow[t]{3}{*}{0.010} \\
\hline $38.5-38.9 \square c$ & 47(11.1) & $15(31.9)$ & $32(68.1)$ & \\
\hline $39-41.2 \square c$ & 71(16.7) & $37(52.1)$ & $34(47.9)$ & \\
\hline
\end{tabular}

\section{Figures}




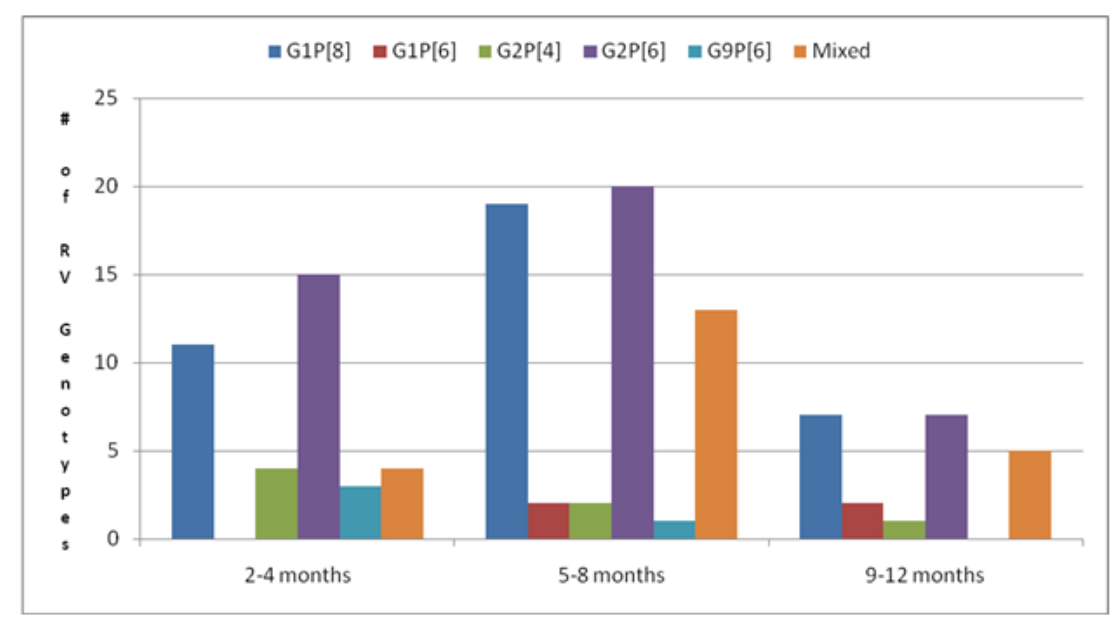

\section{Figure 1}

Age distribution of group A rotavirus genotypes which infected infants who presented with acute diarrhoea at the UTHs Children`s Hospital in 2016.

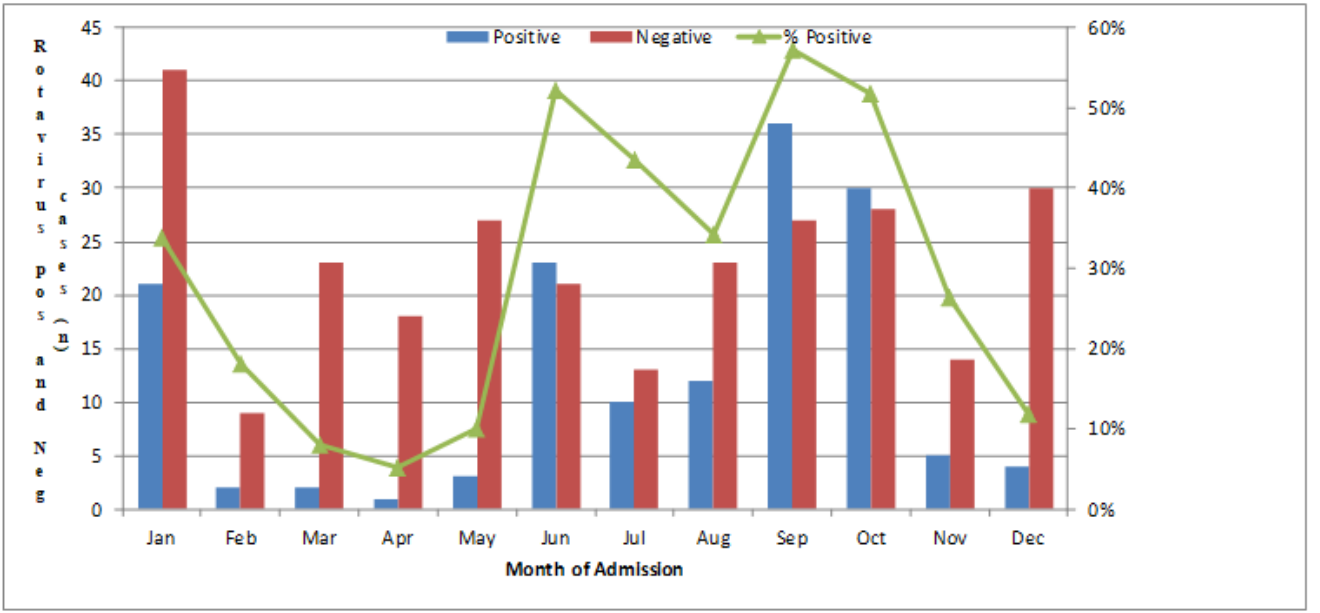

\section{Figure 2}

Monthly distribution Rotavirus infections detected in infants who presented with acute diarrhoea at UTHs Children`s Hospital in 2016.

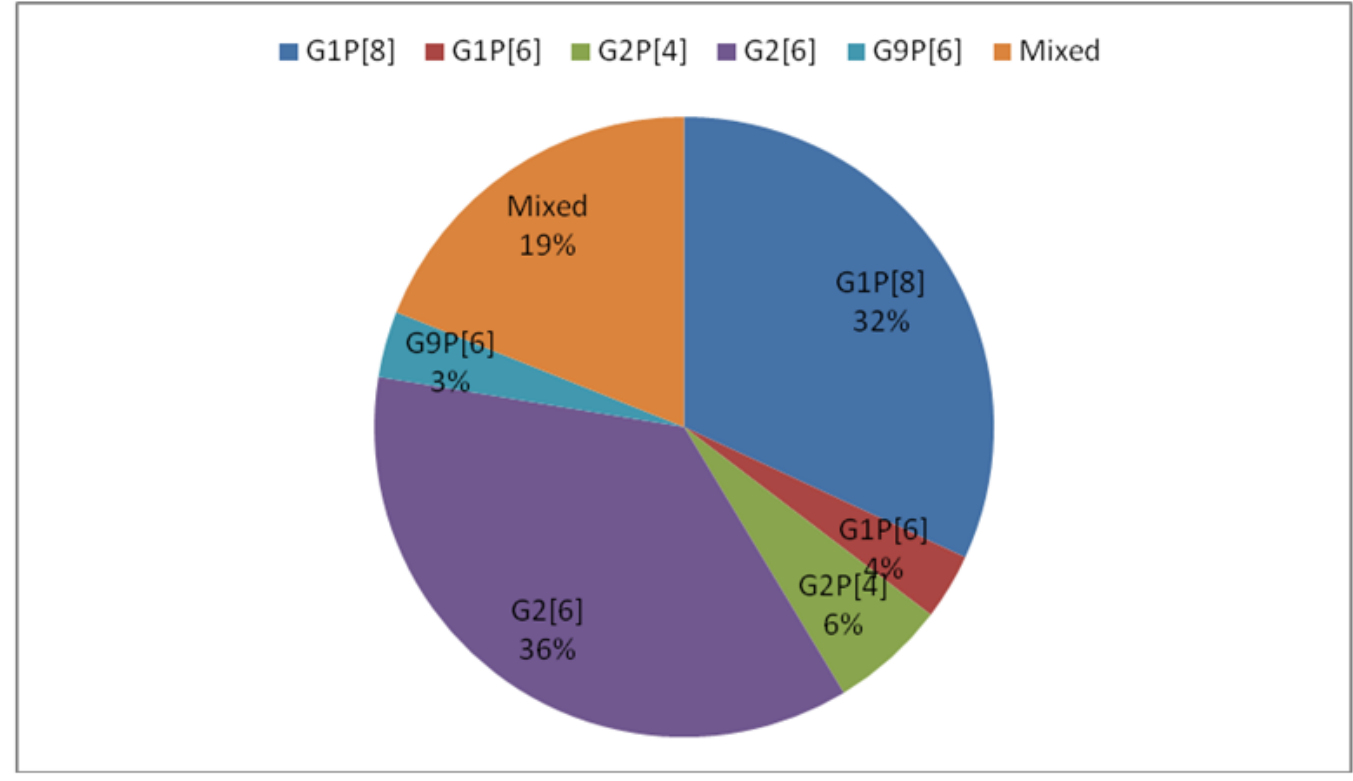

Figure 3

Page 9/10 
Rotavirus genotypes which were responsible for the acute diarrhoea in infants aged 2-12 months at UTHs Children's Hospital in 2016.

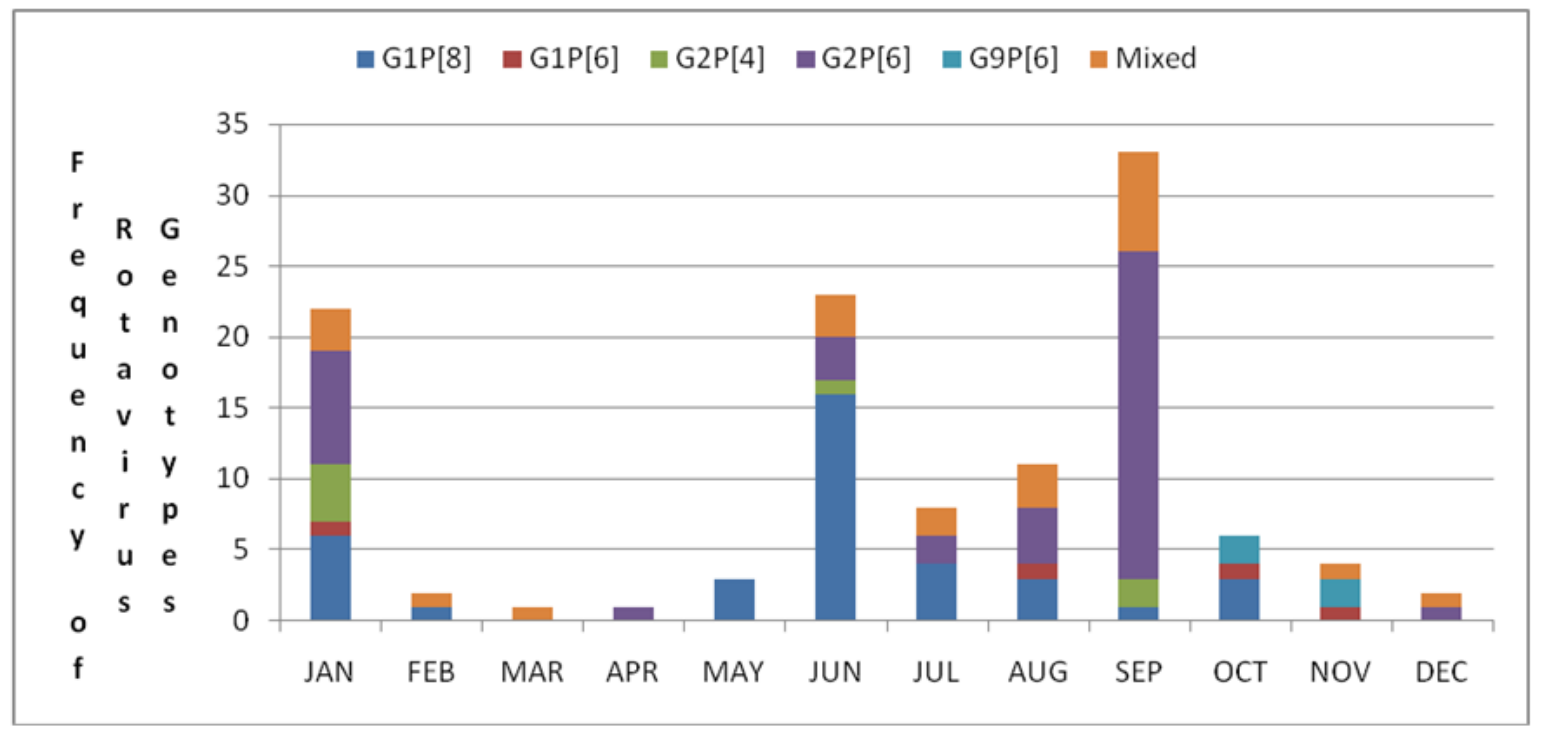

Figure 4

Monthly variation of acute diarrhoea infections which occured at UTHs Children's Hospital in 2016 due to different rotavirus strains

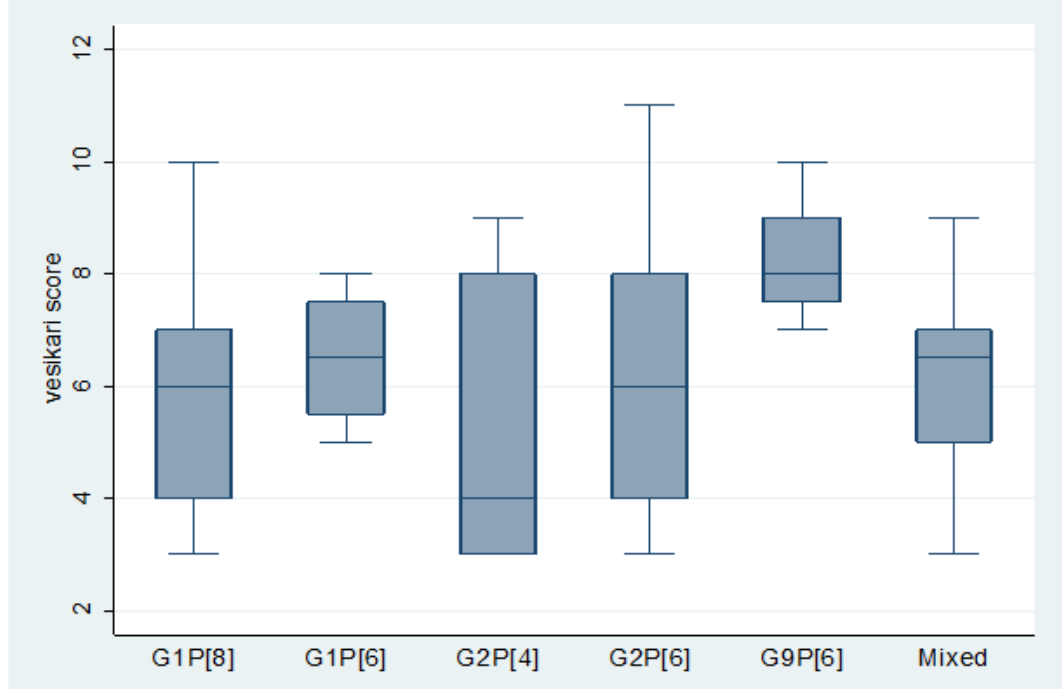

Figure 5

The relationship between the rotavirus strains that caused acute diarrhoea and its severity 